\title{
Design and Characterization of a Fabric-based Softness Display
}

\author{
Matteo Bianchi, Member, IEEE, and Alessandro Serio, Member, IEEE,
}

\begin{abstract}
To enable a realistic tactile interaction with remote or virtual objects, softness information represents a fundamental property to be rendered via haptic devices. What is challenging is to reduce the complexity of such an information as it arises from contact mechanics and to find suitable simplifications that can lead an effective development of softness displays. A possible approach is to surrogate detailed tactile cues with information on the rate of spread of the contact area between the object and the finger as the contact force increases, i.e. force/area relation. This paradigm is called Contact Area Spread Rate. In this paper we discuss how such a paradigm has inspired the design of a tactile device (hereinafter referred to as Fabric Yielding Display, FYD-2), which exploits the elasticity of a fabric to mimic different levels of stiffness, while the contact area on the finger indenting the fabric is measured. In this manner, the FYD-2 can be controlled to reproduce force-area characteristics. In this work, we describe the FYD-2 architecture and report a psychophysical characterization. FYD-2 is shown to be able to accurately reproduce force-area curves of typical objects and to enable a reliable softness discrimination in human users.
\end{abstract}

Index Terms-Tactile display, Softness rendering, Contact area, Human experiments

\section{INTRODUCTION}

Softness, i.e. the subjective measurement of object compliance, plays a relevant role in everyday life activities, from simple tasks such as determining the ripeness of a fruit or the level of air in a bike tire [1] to complex medical procedures such as palpation, where physicians exploit the differences in the perceived hardness of the biological tissues to diagnose the symptoms of some diseases [2].

Although different signals from other sense modalities can provide information about compliance, e.g. auditory [3] or visual cues [4], softness is basically a touch-related property, representing one of the most accessible sources of information after the initial phases of contact [5] and providing fast perceptual cues strictly related to the semantic representation of objects [6]. This fact motivates the attention to the investigation of haptic perception of compliance (e.g. [7], [8], [9], [1]) and the effort in developing effective haptic softness displays in applications such as tele-operation [10] and robot-assisted minimally invasive surgery [11] - where haptic information is mandatory to enable a realistic and compelling human-robot interaction.

Considering the two main channels of haptic sensing (cutaneous cues and kinaesthesia), cutaneous information was shown to be both necessary and sufficient for softness discrimination of compliant objects with deformable surfaces [9], although both types of information are necessary for softness discrimination of compliant objects without deformable surfaces. This cutaneous predominance of cutaneous channels was demonstrated also in the artificial side, where tactile dis-

- Authors are with the Centro di Ricerca "E. Piaggio", Universitá di Pisa, Largo Lucio Lazzarino 1, 56126 Pisa, Italy. M. Bianchi is also with the Dept. of Advanced Robotics, Istituto Italiano di Tecnologia, Via Morego 30, 16163, Genoa, Italy. E-mails: \{matteo.bianchi\}@iit.it, \{alessandro.serio\}@centropiaggio.unipi.it.

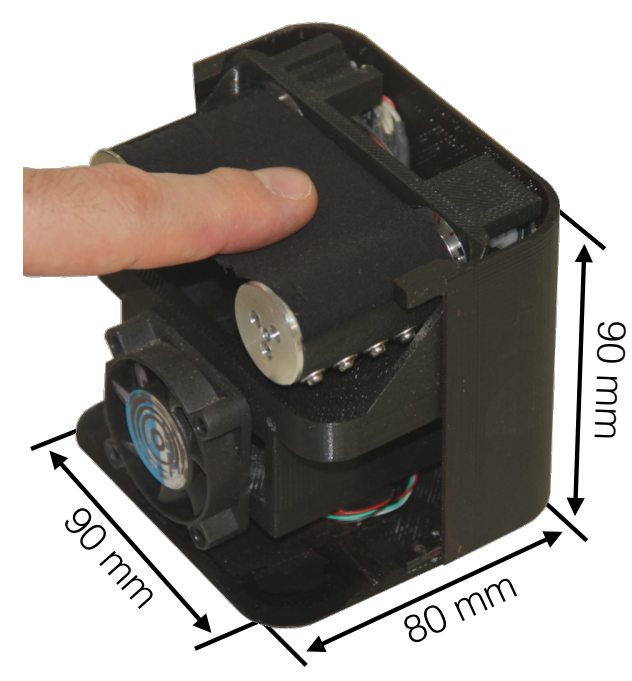

Fig. 1: A subject fingertip interacting with the FYD-2.

plays were proven to elicit a more realistic softness perception than their kinaesthetic counterparts [10], [12].

In literature, different tactile displays have been proposed [13], driven by a range of actuation technologies and mechanisms, including high polymer gel actuators [14], electrorheological [15] and magnetorheological fluids [16], pneumatic arrays [17] and manipulators [18], suction pressure stimuli [19]. In [11] authors presented a tactile device, which exploits two small tilting plates to reproduce surface deformations of a compliant object, while kinaesthetic information is rendered through a modified haptic force feedback device. In [12] authors pioneered the integration of a tactile and kinaesthetic haptic system for softness rendering, showing that softness perception improves when both the modalities are exploited and independently controlled.

Despite the large number of technological solutions, what 
is challenging is trying to harness the complexity of the highdimensional tactile information, arisen from the mechanics of touch, by means of suitable low-dimensional approximations, which can drive design compromises between feasibility, costs and quality of haptic stimuli to be rendered. This approach is further motivated by the fact that even if the number of coordinates needed to completely describe under a mechanical point of view common haptic interactions is larger than three or four, human touch-related experience seems to take place in a lower dimensional space; i.e. nervous system produces nearly instantaneous reductions of dimensions, to convert a complex problem into a manageable set of computational tasks [20]. By adopting the naming used in motor control studies, these low dimensional reductions and simplifications were defined as haptic synergies in [21], [22].

Contact area control can represent a useful and informative reduction of dynamic, force-varying tactile information involved in softness perception, and it has been used to drive the design of several softness displays [10], [23], [24], [25].

In [10] such force-area relationship was defined as the Contact Area Spread Rate (CASR) paradigm and used to develop a pneumatic softness display with hollow cylinders in telescopic arrangement. In [23], a softness hydraulic display was proposed, which controls the fingertip contact area dynamically according to the detected contact force and based on the numerical models of the materials to be rendered. In [24] the softness presentation technique proposed in [23] was integrated with an electrostatic tactile display that can generate different surface texture stimuli.

Inspired by [24], in [25] a softness display, which consists of a silicone rubber sheet placed on a rigid plate, was introduced. Both ends of the sheet are attached to a plastic fork, whose height is controlled via a DC motor: by changing the height, the sheet wraps around the finger, thus resulting in a change of the contact width. In tele-operation tasks, the display was also used in conjunction with a soft sensor, which is capable to optically measure the width of contact area at the remote site, while it is pushed against the remote surface with a given force.

In successive versions focusing on lump detection tasks, the display was endowed with two DC motors, which independently control both sides of the contact area [26], thus enabling asymmetric contact area rendering: softness sensation was hence conveyed by changing both the tension of the flexible sheet and the contact area between the sheet and a fingertip, thus reproducing sensations of soft objects containing lumps beneath their surfaces [27].

Although promising, the practical usage of these systems is however limited due to one or more of the following aspects: (i) the lack of real-time contact area measurement, which severely affects the reliability in reproducing force-area curves, by introducing edge effects and discretization as in [10] or limiting the control of the fingertip contact area only to a finite set of constructed and stored in advance material numerical models as in [23]; (ii) the lack of accuracy in contact area reproduction as in [25]; (ii) constraints in the workspace and integrability issues (especially in robot-assisted minimally invasive surgery applications [28]), which can dramatically reduce the applicability of the displays that perform contact area control by acting on the vertical direction, i.e. by suitably varying their height [26], [27].

To try to overcome these limitations, we propose a fabricyielding display (hereinafter referred to as FYD-2), which exploits the elasticity of a fabric to convey tactual information, providing, at the same time, an on-line measurement of the contact area of the fingertip/object pair by using a web camera placed just beneath the fabric. The device we present in this paper, inspired by other fabric-based softness displays we previously described in [29], [30], exhibits: reduced dimensions (see fig. 1) and a softness control strategy, which can fulfill workspace constraints, also enabling possible integrations with other devices and systems (as shown in the application described in [31], see Subsection 4.3); an actuation system based on two fast motors and an effective sensorization scheme, which consists of a web camera and a force sensor mounted at the base of the device to record the normal contact force exchanged between the finger pad and the fabric. In this manner, the here proposed actuation and sensorization schemes implement a closed-loop control, which allows to track arbitrary force-area characteristics of real specimens. Finally, the actuation scheme also endows the system with an additional degree of freedom, which can be used to convey supplementary haptic cues, such as directional information, for a more compelling and immersive haptic experience.

The paper is partly based on and further extends the work described in [32] and [33], with a more in depth validation of the contact area measurement algorithm and the performance of the system in terms of softness rendering. In the experimental session we describe how we characterized different silicone specimens through a suitably conceived apparatus, which is endowed with an indenter and it is able to control and measure both the force and the area at the contact with the fabric. Experiments where the FYD-2 is used to mimic the experimentally obtained softness and force-area profiles are reported, showing the effectiveness of the here proposed device. Finally a major novel contribution of this paper is a thorough psychophysical characterization of the FYD-2, including softness discrimination experiments with both artificially rendered and real specimens.

\section{System Architecture}

In this section we describe the FYD-2 architecture under a mechanical and control point of view, illustrating how we characterized the device behavior in terms of force-area and force-indentation relationships, while an indenter probes for softness the surface of the display. To achieve this goal, we developed a suitably characterization system. Finally, the contact area measurement technique used with the FYD-2 is reported and validated.

\subsection{Mechanical Description}

The surface of the FYD-2 consists of a layer of isotropic elastic fabric, Superbiflex HN by Mectex S.P.A (Erba, Como, Italy) [30], which exhibits a good resistance to traction. By suitably changing the stretching state of the fabric, subjects 


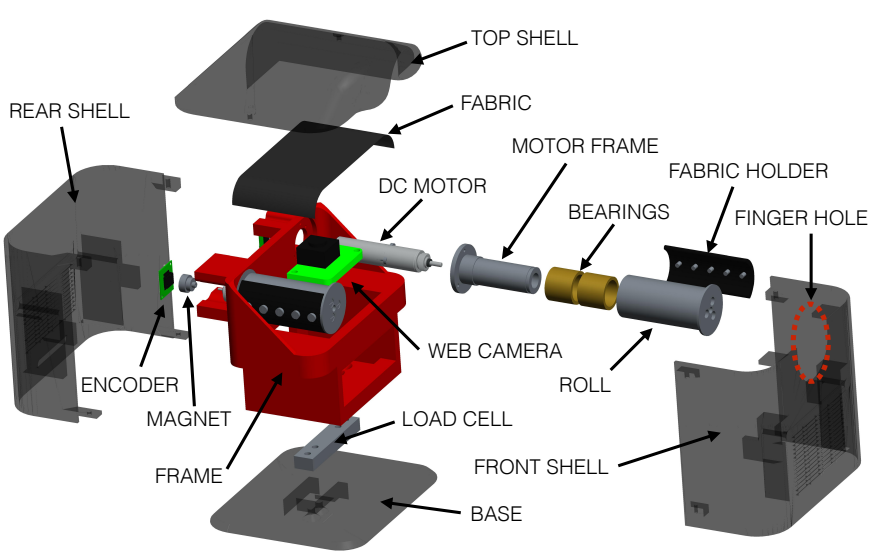

Fig. 2: Exploded drawing of the FYD-2.

interacting with the fabric surface of the device were provided with different levels of stiffness

More specifically, the extremities of a rectangular strip of the elastic fabric $(100 \mathrm{~mm} \times 70 \mathrm{~mm})$ are connected to two rollers, each of them is independently moved by a pulley placed on a motor shaft. Motors are DC Maxon Motor REmax (256:1, 3 Watt) by Maxon Motor ag (Sachseln, Switzerland). We chose these motors since they provide a good trade-off between velocity and torque, which is necessary to enable fast changes of the stretching state of the fabric.

DC Motors are inserted into the rollers, while a custom made electronic board (PSoC-based electronic board with RS485 communication protocol) controls motor positions on the basis of the readings of two absolute magnetic encoders (12 bit magnetic encoder by Austria Microsystems - Unterpremstaetten, Austria - AS5045, with a resolution of $0.0875^{\circ}$ ).

An exploded drawing of the system is shown in fig. 2. The dimensions of the FYD-2 are $90 \mathrm{~mm}$ (height) $\times 90 \mathrm{~mm}$ (length) $\times 80 \mathrm{~mm}$ (width), less than one third of the dimensions of the previous version of the fabric-based softness display [30] (see also fig. 1 for more details). This reduction of dimensions might be exploited to integrate the FYD-2 with other systems (e.g. to decouple kinaesthetic and cutaneous cues as proposed in [12]), as it was done in [31], where the FYD-2 was integrated into an experimental apparatus used to investigate the role of contact area change over the finger pad on the perception of finger displacement (see Subsection 4.3).

As previously said, a given level of softness is associated to a stretching state of the fabric and it can be achieved by suitably controlling the two motors (motor 1 and motor 2). More specifically, when motor 1 rotates in a counter-clockwise direction and motor 2 rotates in a clockwise direction they stretch the fabric, which increases its stiffness. On the other hand, when motor 1 rotates in a clockwise direction and motor 2 rotates in a counter-clockwise direction, they relax the fabric which becomes softer, see fig. 3 .

An additional "translational" degree of freedom can be obtained when motors rotate in the same direction: in this case the finger interacting with the fabric can be moved left and right. However this additional degree of freedom, although promising in enhancing the immersiveness of the reproduced haptic experience, is still under investigation.

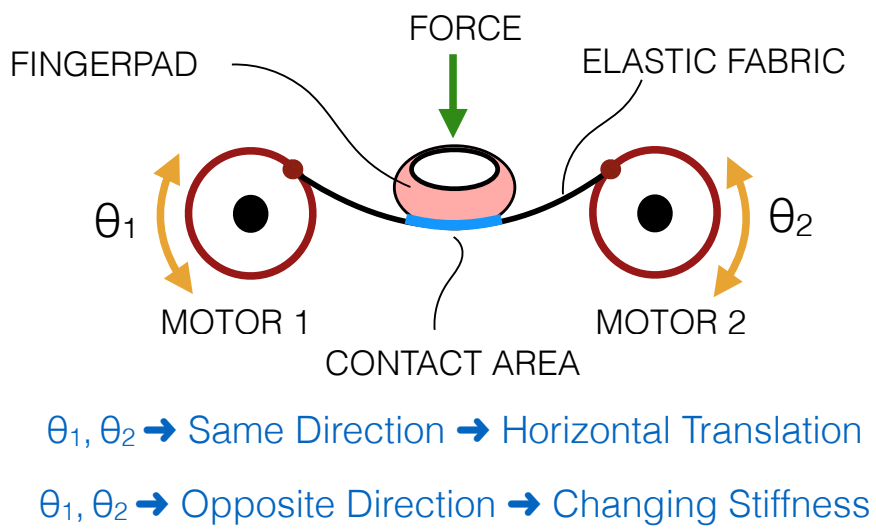

Fig. 3: Working principle of the FYD-2: when motor 1 rotates in a counter-clockwise direction and motor 2 rotates in a clockwise direction, they stretch the fabric, thus increasing its stiffness. On the other hand, when motor 1 rotates in a clockwise direction and motor 2 rotates in a counter-clockwise direction, they relax the fabric which is felt softer. An additional "translational" degree of freedom can be obtained when motors rotate in the same direction. Notice that the rotation angle for motor $1\left(\theta_{1}\right)$ is positive if motor 1 counterclockwise rotates; on the contrary, the rotation angle for motor $2\left(\theta_{2}\right)$ is positive if motor 2 clockwise rotates.

FYD-2 is also capable to on-line measure the contact area between the finger pad and the fabric. To achieve this goal, we placed just beneath the fabric $(30 \mathrm{~mm})$ (see fig. 2) a web camera (Microsoft "LifeCam HD-3000" with a resolution of $640 \times 480 @ 30 \mathrm{fps})$, with vertical focal axis, and two high luminosity Light Emission Diodes (LEDs), whose luminosity can be regulated with a trimmer. The segmentation algorithm used to estimate the contact area is based on binarization thresholds, which were heuristically calculated considering the difference between background luminosity and contact area luminosity, as it was described in [30]. An hemispherical cover was placed on the device to guarantee uniform and reproducible luminosity conditions through successive haptic interactions. A validation of the algorithm and a mathematical model to compute the contact area are described in Subsection 2.3.

The FYD-2 is also endowed with a load cell (Micro Load Cell, 0 to 780 g, - CZL616C - from Phidgets (Calgary, AB Canada)) placed at the base of the device, to record the normal force exerted by the finger interacting with the fabric. As it will be discussed later in the text, FYD-2 can provide also the measurement of indentation, on the basis of the measurement of contact area and characterization data (see Section 2.2).

All the structural parts of FYD-2 are in ABSplus - Stratasys, rapid prototyping material, while the rollers are in aluminium and they were fabricated using a computer numeric control machine.

\subsection{Characterization}

To correctly use FYD-2, we needed to first characterize its mechanical behavior and workspace, i.e. the stiffness levels and range that can be achieved for different stretching states of the fabric, w.r.t the two haptic modalities involved in softness perception (and haptic interaction more in general), namely, ki- 


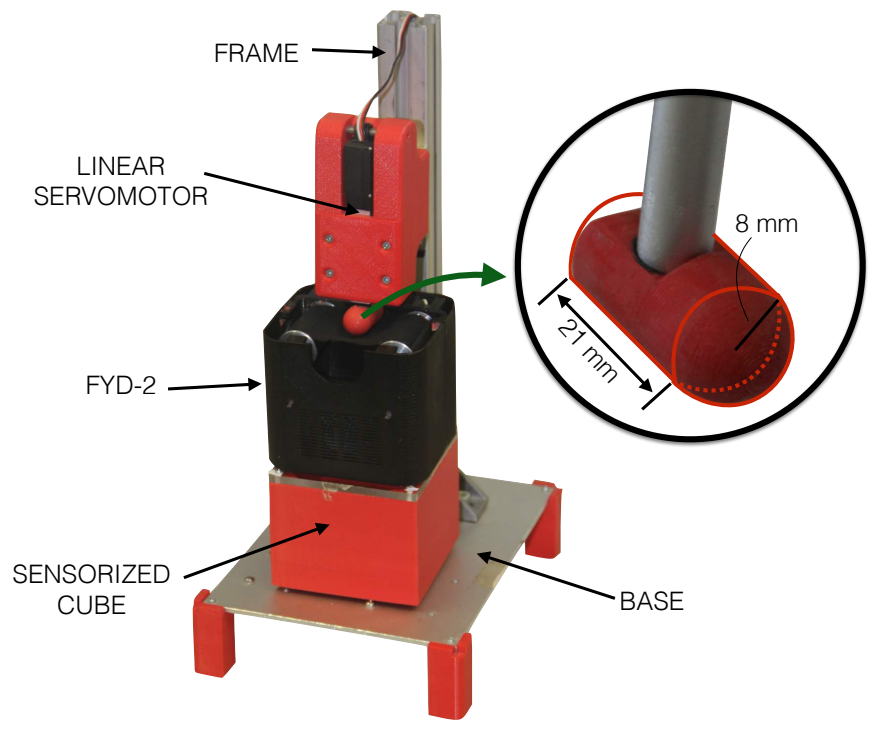

Fig. 4: The indenter used to characterize the FYD-2 and the silicone specimens. In the sensorized cube we placed a webcam and a load cell.

naesthetic perception and cutaneous channels. Using simplified mathematical abstractions, we can consider the relationship between the indenting force ( $F$, which we expressed in $[\mathrm{N}]$ ) and the overall rigid displacement (or indentation) between the two bodies $(\delta$, which we expressed in $[\mathrm{mm}])$, i.e. $F(\delta)$ curve as it results from the interaction of the finger with the surface of an object, as an approximation of the kinaesthetic information involved in softness perception. At the same time, according to CASR paradigm [10], a large part of the cutaneous information involved in softness perception can be approximated with the relationship between the indenting force and the contact area ( $A$ expressed in $\left.\left[\mathrm{mm}^{2}\right]\right)$ on the finger pad arisen at the contact with the object, i.e. the $F(A)$ curve.

Following these assumptions [12], we obtained the $F(\delta)$ and $F(A)$ curves for different stretching states of the fabric. More specifically, these stretching states correspond to different angular positions of motor 1 and motor $2\left(\theta_{1}\right.$ and $\theta_{2}$, respectively), from $0^{\circ}$ to $80^{\circ}$, with an incremental step of $10^{\circ}$ (see fig. 3). Notice that the sign of $\theta_{1}$ angle is positive when motor 1 counterclockwise rotates, while the sign of $\theta_{2}$ is positive when motor 2 clockwise rotates.

To achieve the characterization curves, we used a custom made testing system to compress the fabric, see also figs. 4 and 7. Briefly, the system consists of an indenter attached to the shaft of a servo-controlled linear actuator (Firgelli L12-50-100-6-L, by Firgelli Technologies Inc. (Victoria, BC Canada)). The indentation is directly controlled by the motor, while the contact force and the contact area are measured by FYD-2 through the web camera and load cell, respectively. The procedure for contact area measurement is based on luminosity binarization thresholds and it is analogous to the one reported in [34]. For a complete description of the characterization system, please refer to Section 3 . The indenter was moved using fixed indentation steps of $1 \mathrm{~mm}$ each, for an overall displacement of $20 \mathrm{~mm}$ with an indenting velocity of (a) Fabric F(A) Characterization

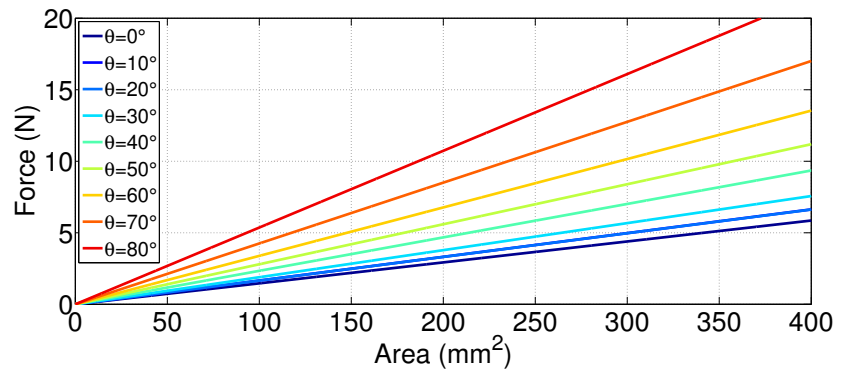

(b) Fabric $\mathrm{F}(\boldsymbol{\delta})$ Characterization

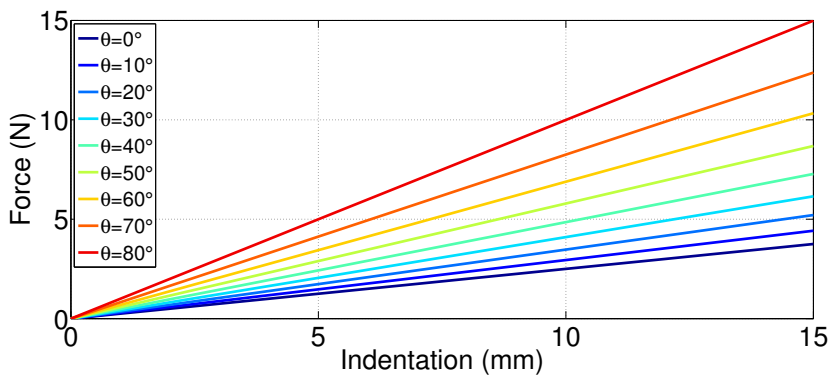

Fig. 5: (a): F(A) curves of FYD-2 at different motor positions; (b): $\mathrm{F}(\boldsymbol{\delta})$ curves of FYD-2 at different motor positions.

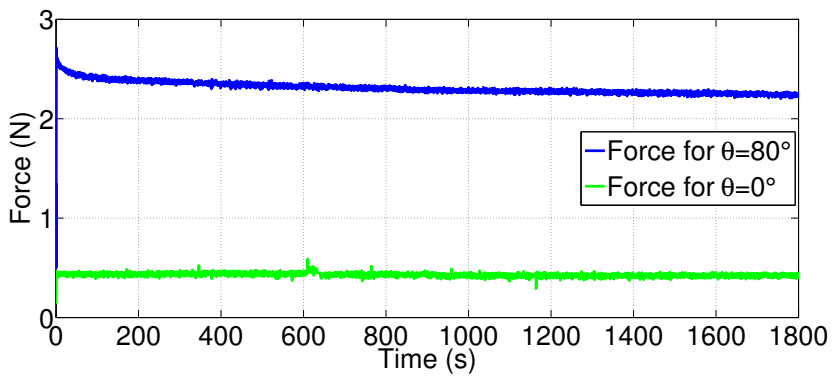

Fig. 6: Stress-Relaxation test of the fabric used in the FYD-2 for $\theta=0^{\circ}$ and for $\theta=80^{\circ}$.

$5 \mathrm{~mm} / \mathrm{s}$. The range of the contact force varies from 0 to $20 \mathrm{~N}$.

The indenter consists of a cylinder (with radius $r$ equal to 8 $\mathrm{mm}$ and length $L$ equal to $21 \mathrm{~mm}$ ) and a semi-sphere of same radius fabricated in ABSplus - Stratasys (rapid prototyping material), and it models the last phalanx of a human finger, see figs. 4 and 7. We chose this value for $r$ since it is coherent with typical values of human finger diameter [35]. Ideally, the differences between the indenter (which is a non-compliant object) and human fingertip (which is a compliant object) should be considered during the characterization procedure; however, since the deformation of the fingertip that interacts with the fabric is small, the approximation of the indenter with a non-deformable object is still acceptable. Indeed, given the range of stiffness that can be reproduced by the device (maximum stiffness $2 \mathrm{~N} / \mathrm{mm}$, in the range of contact force values from 0 to $20 \mathrm{~N}$ ), the deformation of the fabric is usually larger than the one produced on the finger pad (as it results also considering its mechanical properties known from literature [36]).

In order to always provide users with same stiffness charac- 
teristics over different interaction trials, we need to take into account possible changes of the stiffness distribution on the fabric from center to edges. To prevent such a possibility, we individuated a square interaction area at the center of the fabric with length $300 \mathrm{~mm}$, where the stiffness distribution of the fabric does not change. Such interaction area was also used to identify a region of interest (ROI) to remove any edge effect in the measurements of the web cam (see Subsection 2.3). We can constraint users to touch the fabric in the interaction area by allowing them to interact with the FYD-2 surface only through the hole placed on the front shell of the external shield (see fig. 2), which is centered on the ROI.

We used different polynomial fitting procedures to interpolate the force/indentation curves of the fabric at different motor positions. Although cubic interpolation provides the best numerical results, since there is a slight deviation from linearity for high values of indentation $(\delta>10 \mathrm{~mm})$, it is reasonable to assume the properties of the fabric to be linear elastic over different motor positions (goodness of fit: $\left.R^{2}>0.93\right)$ and for the average range of indentation $(\delta \leq 10$ $\mathrm{mm})$, as it results from the experiments with humans. In other terms, $F=\sigma(\theta) \delta$, where $\sigma$ in $[\mathrm{N} / \mathrm{mm}]$ is the stiffness coefficient at motor position $\theta$ in $\left[{ }^{\circ}\right]$. Characterization results for different motor positions are reported in fig. 5(b).

In this manner, it is also possible to achieve an on line reconstruction of the indentation, based on the measured force and motor position.

$$
\delta=\frac{F}{\sigma}
$$

Moreover, we also interpolated the fabric stiffness $\sigma$ w.r.t the motor position $\left(R^{2}=0.94\right)$ in order to build a complete stiffness map

$$
\sigma(\theta)=0.09 \theta+0.1795 \text {. }
$$

Coherently, the force/area curves interpolated at fixed motor positions are linear, i.e. $F=v(\theta) A\left(R^{2}>0.93\right)$, where $v$ in $\left[\mathrm{N} / \mathrm{mm}^{2}\right]$ is the linear interpolation coefficient of the force/area curves, see fig. 5(a).

At the same time, we performed a stress relaxation test to analyze and take into account possible viscoelastic properties of the fabric. We used the previously described characterization system (see fig. 4) to indent the fabric at $5 \mathrm{~mm}$ for different motor positions, i.e. stretching states of the fabric. We recorded the values of force for $1800 \mathrm{~s}$ to verify the presence of possible force relaxation phenomena. Results are reported in fig. 6. As it is noticeable, a relaxation is present, especially for higher fabric stretching states (i.e. $\theta=80^{\circ}$ ). Interpolating such relaxation phase assuming an exponential decay $\left(R^{2}>0.88\right)$, we achieved a time constant $\tau>4 \times 10^{5}$ s. Since $\tau$ is much more higher than the average interaction time of the human finger during the experiments (around $10 \mathrm{~s}$ ), we decided to disregard viscoelastic effects of the fabric, since they are out of the aim of this paper, and to investigate them in future works.

\subsection{Contact Area Measurement}

In order to suitably replicate force-area CASR curves arisen from the interaction of the finger with common objects,

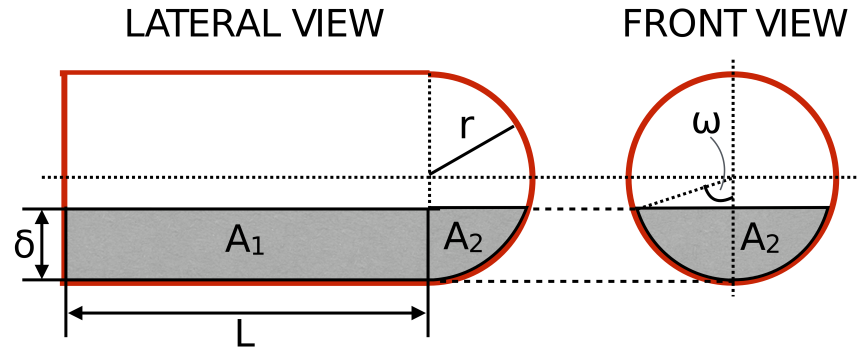

Fig. 7: The indenter: sketch and main geometric features $(r=8 \mathrm{~mm}$, $L=20 \mathrm{~mm}$ ). $\delta$ indicates the indentation produced by the indenter on the fabric surface. The shadow parts belong to the contact area of the indenter with the fabric.

FYD-2 needs to control and hence measure the area at the contact between the finger and the fabric. To do this we proposed a suitable segmentation algorithm [29]. This algorithm is based on the binarization of the image acquired through the camera. To avoid a too high computational workload and hence assuring a fast processing, we used only one image band (the $\mathrm{R}$ band, which can be represented as a $320 \times 240$ matrix of integer numbers) in the area detection algorithm. The leading idea of the contact area algorithm is as it follows: while the fabric is probed for softness by the finger, the indented surface of the fabric is closer to the camera with respect to the outer region. Consequently, this area will be more lighted up by the LEDs. The difference between background luminosity and contact area luminosity is discriminated via binarization thresholds, which are heuristically calculated. In this manner, the pixels in the image belonging to the contact area can be individuated and displayed (see also [30]). We calculated the binarization thresholds for different angular positions of motor 1 and $2(\theta$ $=\theta_{1}=\theta_{2}$ ). As previously mentioned, in order to guarantee uniform and repeatable luminosity conditions, we placed a cover on the top of the external shell of the device. The measured contact area $A_{\text {me }}$ expressed in $\left[\mathrm{mm}^{2}\right]$ is estimated as

$$
A_{\mathrm{me}}=N_{c} \frac{A_{f}}{N_{w}},
$$

where $N_{c}$ is the number of pixels belonging to the contact area; $A_{f}$ is the area captured by the web camera (frame area) in $\left[\mathrm{mm}^{2}\right]$ and $N_{w}$ is the web camera resolution (i.e. $320 \times$ 240 pixels).

However, we need to correct this value $A_{\text {me }}$ in order to take into account the bias due to the varying distance of the fabric from the camera focus and to remove any lens distortion (notice that edge effects such as vignetting are also removed by individuating a ROI, as it is described in Section 2.2). In other terms: while the finger indents the fabric, the fabric will be closer to the camera focus (which is fixed) and hence the image of the fabric "seen" by the camera will be greater w.r.t the real one (as it can be well explained through the optic flow model [37]). The correction factor $K$ can be defined as the ratio between the theoretic contact area $A_{t}$, which can be obtained knowing the geometry of the indenter (see fig. 7) and 
the measured indentation as

$$
A_{t}=A_{1}+A_{2}=2 L r \cos ^{-1}\left(\frac{r-\delta}{r}\right)+\pi \delta r,
$$

where $r$ and $L$ are the radius and the length of the cylinder, respectively (in $[\mathrm{mm}]$ ), $A_{1}$ is the area of the cylinder in contact with the fabric, $A_{2}$ is the area of the semi-sphere in contact with the fabric (both in $\left[\mathrm{mm}^{2}\right]$ ) and $\omega=\frac{r-\delta}{r}$ (see also figs. 4 and 7 for more details). Hence, it is possible to compute the correction factor $(K)$ as

$$
K=\frac{A_{t}}{A_{m e}} .
$$

The measured area corrected with $K$ will be $A_{m}=K A_{m e}$. We computed the correction factor for different angular motor positions (from $0^{\circ}$ to $80^{\circ}$ with step of $10^{\circ}$ ) and for different values of indentation $\delta$, ranging from 0 to $20 \mathrm{~mm}$, with incremental step of $1 \mathrm{~mm}$. No difference in $K$ values was found among different motor angular positions.

Afterwards, we derived the relationship between $K$ and the value of indentation $\delta$, being $K(\delta)$, which we approximated with a cubic function $\left(R^{2}=0.92\right)$

$$
\begin{aligned}
K(\delta)= & -\left(2.77 \cdot 10^{-5}\right) \delta^{3}+\left(6.939 \cdot 10^{-3}\right) \delta^{2} \\
& -0.2639 \delta+3.781
\end{aligned}
$$

For $\delta>20 \mathrm{~mm}, K$ value is constant and equal to 1.0567 .

\section{Softness Rendering}

As already said, since softness perception relies on both kinaesthesia and cutaneous information, FYD-2 can be controlled to track both $F(\delta)$ or $F(A)$ curves. Of course both characteristics are strictly related each other in the device.

However, such a coupling is not unique. Indeed, it is possible to find two specimens with: (i) different force-area and same force-indentation curve, i.e. one should rely only on cutaneous information to discriminate the specimens, while kinaesthetic information is kept unchanged; (ii) different forceindentation and same force-area curve, i.e. one should rely only on kinaesthetic information to discriminate the specimens and cutaneous information is kept unchanged; (iii) different force-area and different force-indentation curves, i.e. full integrated (kinaesthetic and cutaneous) haptic information is available for softness discrimination.

Examples of such cases and a decoupled strategy to independently control the characteristics $F(\delta)$ and $F(A)$ to render specimens in (iii) are deeply discussed in [12], where it was also confirmed that cutaneous information is predominant for softness discrimination (i.e. performance in softness discrimination for specimens in (i) is better than for specimens in (ii)).

With this as motivation, our main interest is in tracking the $F(A)$ characteristic of a specimen in order to render its softness (indeed, CASR paradigm was the leading idea for the development of FYD-2).

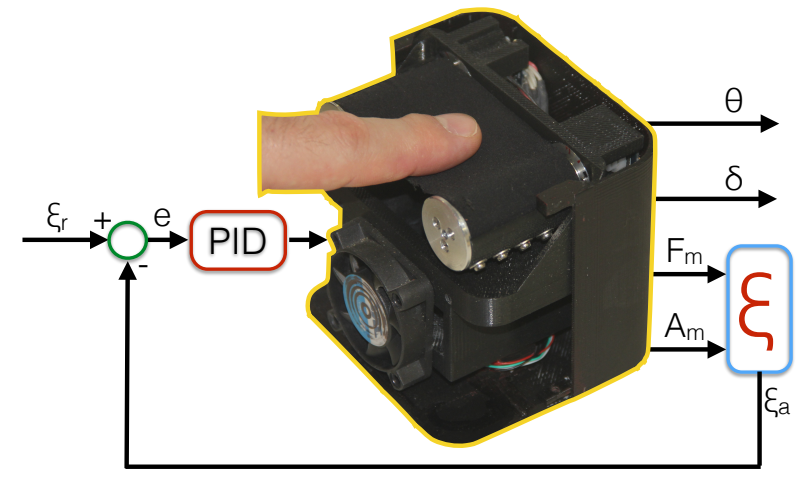

Fig. 8: Control scheme for $F(A)$ tracking.

However, for the sake of completeness, we also report the control scheme and an example of the $F(\delta)$ tracking performance of the device. Notice that when the system behaves like a $F(\delta)$ tracker, i.e. to mimic a given stiffness, the behavior is analogous to the one exhibited by common kinaesthetic systems (which basically act as force displays [38]), although cutaneous cues are clearly conveyed to subjects via fabric deformation. Furthermore, the on-line measurement of the contact area is now available and it can provide additional tactile information about the haptic interaction in act.

\subsection{Force-Area Rendering}

The $F(A)$ characteristics obtained during the characterization phase are linear at fixed motor positions; therefore, linear $F(A)$ curves can be simply mimicked by using (or interpolating across motor positions) the characterization curves. However, in order to reproduce $F(A)$ characteristics of common objects, which are usually quadratic [10], the position of the motors needs to be controlled and suitably rapidly changed, based on the actual contact area. This fact motivated the need for a fast actuation system. Let be $F(A)=\xi_{r} A_{r}^{2}$, the quadratic curve to be tracked, where $\xi_{r}$ (in $\left[\mathrm{N} / \mathrm{mm}^{4}\right]$ ) is the quadratic coefficient of the area. In order to properly implement the control, we need to know the actual $\xi_{a}\left(\left[\mathrm{~N} / \mathrm{mm}^{4}\right]\right)$ coefficient. This coefficient can be obtained each time by dividing the indenting force $F_{m}$, measured through the load cell, for the squared value of the measured area $A_{m}$. Therefore $\xi$ is computed as

$$
\xi=\frac{F_{m}}{A_{m}^{2}+\sigma}
$$

where $\varepsilon$ is a number $<<1$ and different from 0 , which is used to avoid numerical errors when $A=0$ (which means no contact with the FYD-2 fabric surface; this fact may occur even if the finger is effectively contacting the surface, due to some errors related to the binarization threshold).

PID control is then used to move the motors, based on the error between the reference coefficient $\xi_{r}$ and the actual one $\xi_{a}$ (see fig. 8).

For PID parameter tuning, we chose to use the open loop Ziegler-Nichols rules to individuate starting point values, which were heuristically adjusted to achieve better control results. We obtained the best performance individuating different 
(a) F(A) Tracking

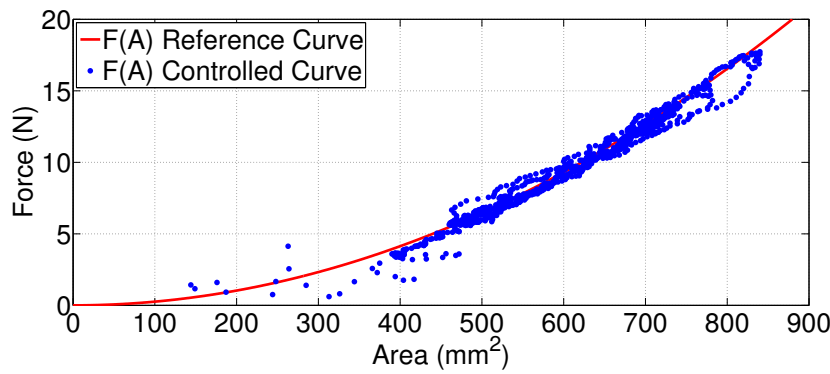

(b) Area Tracking

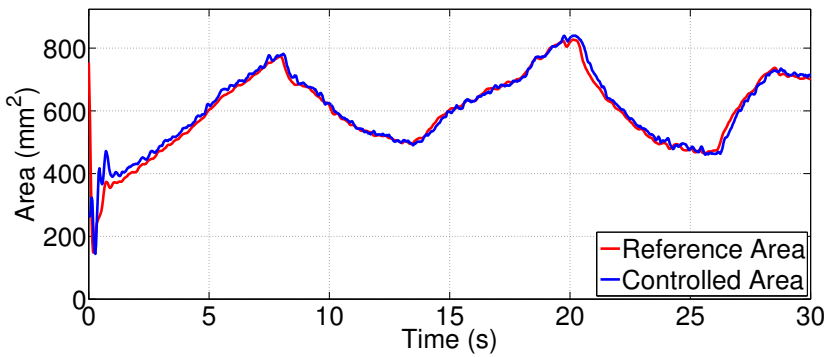

(c) Indentation

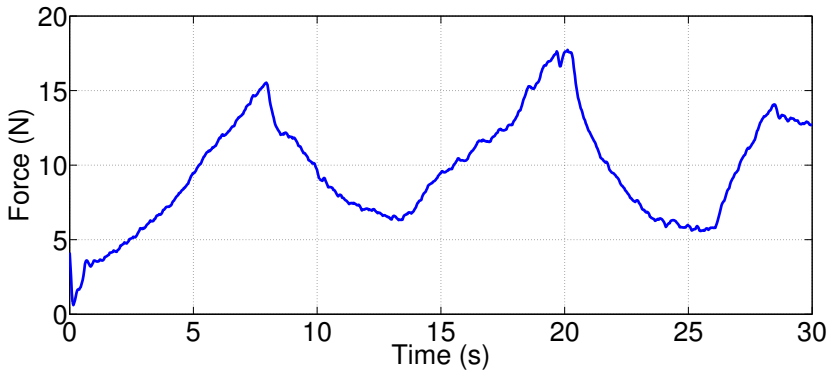

Fig. 9: In the first plot (a) we show the reference $F(A)$ curve (in red), while the pairs (measured controlled area, measured force) are represented as blue dots. In the second plot (b) we report the contact area control (blue line) vs. contact area reference (red line). In the third plot (c) the measured force is reported. These plots refer to the silicone specimen SS1.

ranges for $\xi_{r}$ values and then associating a different parameter set to each range.

We used a sweeping procedure, starting from the lowest value which is possible to simulate, i.e. $\xi_{r_{l o}}=7 \cdot 10^{-6} \mathrm{~N} / \mathrm{mm}^{4}$ to the highest one $\xi_{r_{h i}}=3 \cdot 10^{-5} \mathrm{~N} / \mathrm{mm}^{4}$. The procedure is quite simple: we tuned the parameters and then increase the value of $\xi_{r}$ till the controller started to give bad tracking results. We used this value of $\xi_{r}$ as the starting value for the next range. Then the procedure was repeated up to the biggest reference value that can be simulated.

The obtained ranges with the associated PID constants are

1) $7 \cdot 10^{-6} \mathrm{~N} / \mathrm{mm}^{4} \leq \xi_{r} \leq 1.6 \cdot 10^{-5} \mathrm{~N} / \mathrm{mm}^{4}, P=140, I=$ $3500, D=0$

2) $1.6 \cdot 10^{-5} \mathrm{~N} / \mathrm{mm}^{4}<\xi_{r} \leq 2.2 \cdot 10^{-5} \mathrm{~N} / \mathrm{mm}^{4}, P=100, I=$ $3700, D=0$

3) $2.2 \cdot 10^{-5} \mathrm{~N} / \mathrm{mm}^{4}<\xi_{r} \leq 3 \cdot 10^{-5} \mathrm{~N} / \mathrm{mm}^{4} P=35, I=$ $3800, D=0$.

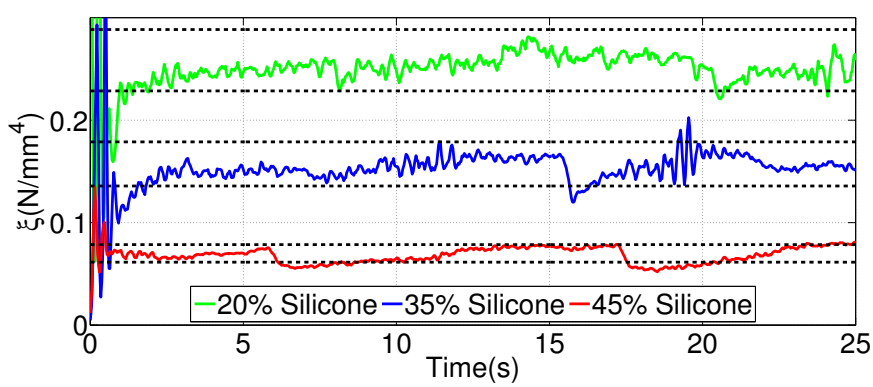

Fig. 10: Control of $\xi_{r}$ for the three silicone specimens, which were used also for the experiments with humans reported in Section 4. The dashed black lines define the $5 \%$ error bounds.

(a) Silicone F(A) Characterization

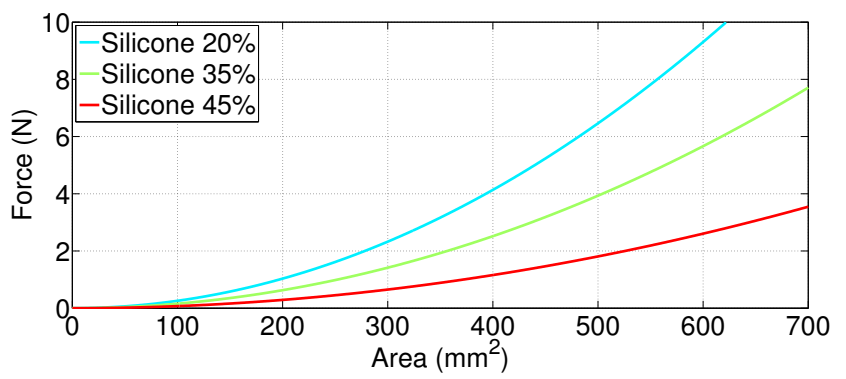

(b) Silicone $\mathrm{F}(\boldsymbol{\delta})$ Characterization

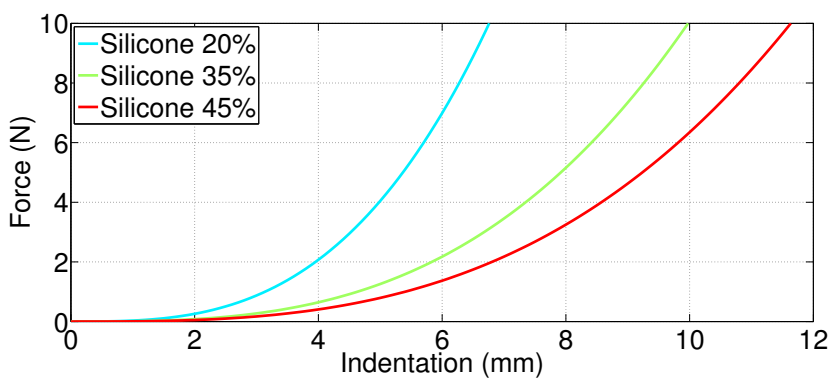

Fig. 11: The $F(A)$ characteristics of the three silicone specimens used for the experiments with humans (see also Section 4). Silicone $20 \%$ corresponds to SS1, while Silicone $35 \%$ and Silicone $45 \%$ can be referred to as SS2 and SS3, respectively.

\subsubsection{Specimen Characterization}

To properly validate the system performance in terms of $F(A)$ tracking, we chose to reproduce the characteristics of three silicone specimens, chosen among the ones reported in [12]. The specimens were half-spheres of radius of $20 \mathrm{~mm}$ and they were made of material obtained by mixing a given quantity of a commercial bicomponent, room temperature-curing silicone (BJB TC-5005A/B), with a variable percentage of plasticizer (BJB TC-5005C), acting as a softener. The amount of softener in the mixture was chosen as $20 \%, 35 \%$ and $45 \%$, referring to the specimen SS1, SS2 and SS3, respectively. We derived the relationship between the contact force and the contact area for SS1, SS2 and SS3 with the characterization system introduced in the Section 2.2 and reported in fig. 4. The specimens to be characterized were placed under the motor stroke and put in progressive contact with the transparent glass of the sensorized cube. The indenting velocity was $5 \mathrm{~mm} / \mathrm{s}$ with step of $1 \mathrm{~mm}$. The contact force measured was within the range of 0.5 to 


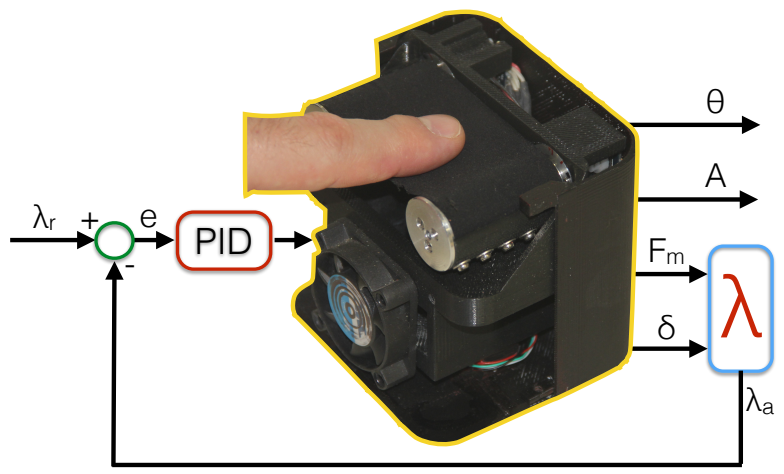

Fig. 12: Control scheme used for force - indentation tracking experiments.

$10 \mathrm{~N}$.

A webcamera - Microsoft LifeCam HD-5000 - was put under the glass. As the indenter pushed against the specimen, the web cam captured a snapshot of the surface flattened against the plexiglass. In order to enhance contours of contact area a thin white paper behaving as optical filter was placed between the specimen and the plexiglass. The procedure is analogous to the one reported in [34].

As previously described, we used heuristic binarization thresholds based on luminosity to obtain the contact area. Furthermore, for each contact area, the indentation force was also measured by means of the load cell placed in the sensorized cube, at the base of the system. In this manner the $F(A)$ and $F(\delta)$ curves were obtained for SS1, SS2 and SS3, see fig. 11. The $F(\delta)$ curves were interpolated using a cubic approximation $\left(R^{2}>0.97\right)$. For further details on the mechanical characteristics of the silicone specimens, the reader is invited to refer to [12].

\subsubsection{System Performance Validation}

The $F(A)$ tracking experiments were performed by a male subject (age 32) touching the fabric with his index finger pad, using a probing frequency of approximately $0.2 \mathrm{~Hz}$.

For the sake of space, we report in fig. 9 only the results of the force-area control for SS1. The RMSEP in terms of $\xi_{r}$ is $5 \%$, while the RMSEP in terms of area tracking is $2.2 \%$.

In fig. 10 , the quadratic coefficients $\xi_{r}$ s relative to $\mathrm{SS} 1$, SS2 and SS3 are reported. They are $2.585 \cdot 10^{-5} \mathrm{~N} / \mathrm{mm}^{4}$, $1.573 \cdot 10^{-5} \mathrm{~N} / \mathrm{mm}^{4}$ and $7.238 \cdot 10^{-6} \mathrm{~N} / \mathrm{mm}^{4}$, respectively (average $R^{2}$ equal to 0.94 ). As it can be seen from fig. 11, we chose these specimens because they are approximately equally spaced in the force-area plane. We controlled FYD-2 in order to reproduce the force-area behavior of the silicone specimens using the control scheme described in Section 3.1.

As it can be seen in fig. 10, FYD-2 can effectively reproduce the three silicones in the $\xi_{r}$ range, with a RMSEP of $5.1 \%, 6.2 \%$ and $9.9 \%$ without overlapping, for SS1, SS2 and $\mathrm{SS} 3$, respectively.

\subsection{Force-Indentation Rendering}

The aim of the experiments here reported is to track given force-indentation curves. Since the characterization curves of (a) Cubic Stiffness Control

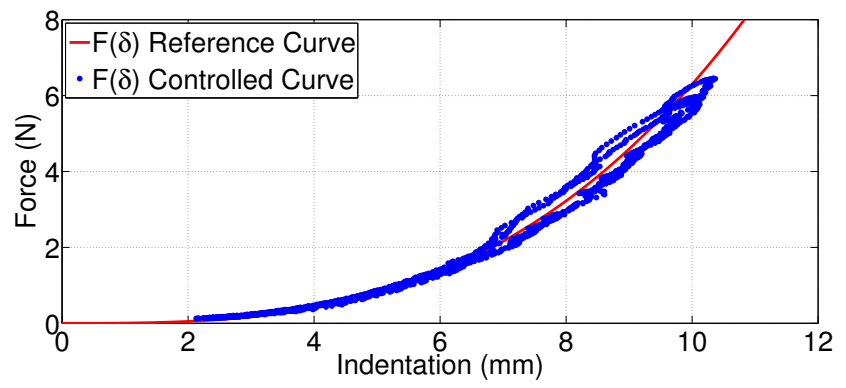

(b) Force

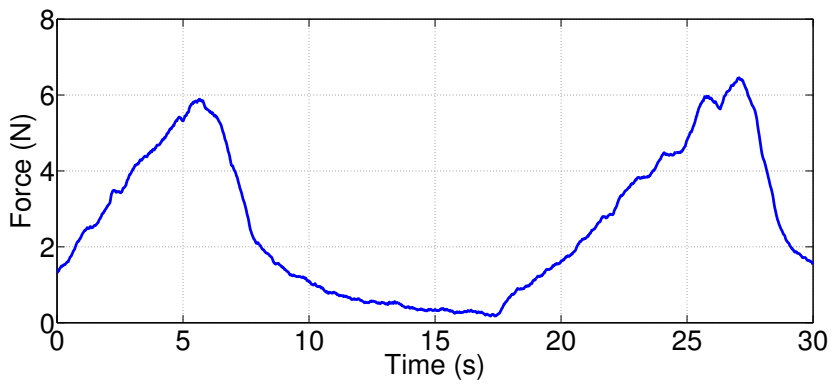

(c) Indentation

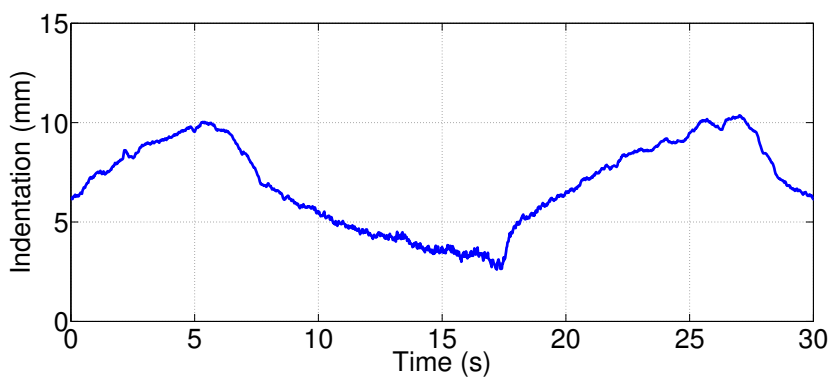

Fig. 13: Tracking of force-indentation characteristic of SS3 and timeevolution of measured force and estimated indentation, respectively.

FYD-2 are linear, linear $F(\delta)$ curves can be simply mimicked by using (or interpolating across motor positions) the characterization curves, as already described in the previous Subsection for the force-area control of linear $F(A)$ curves.

In the experiment of fig. 13 we report the performance of FYD-2 in tracking the force-indentation curve of the silicone specimen SS3. Let be $F(\delta)=\lambda_{r} \delta^{3}$ the force-indentation characteristic for the silicone specimen SS3, where $\lambda_{r}\left(\left[\mathrm{~N} / \mathrm{mm}^{3}\right]\right)$ is the cubic stiffness coefficient of the curve.

Analogously to the control of non linear force-area curves, we need to know the actual $\lambda_{a}\left(\left[\mathrm{~N} / \mathrm{mm}^{3}\right]\right)$ coefficient. This coefficient can be obtained each time by dividing the indenting force $F_{m}$, measured through the load cell, for the third power of the indentation value obtained from the $F(\delta)$ chracterization curves of FYD-2. Therefore $\lambda_{a}$ is computed as

$$
\lambda_{a}=\frac{F_{m}}{\delta^{3}+\varepsilon}
$$

where $\varepsilon$ is a number $<<1$ and different from 0 , which is used to avoid numerical errors.

PID control is then used to move the motors, based on the error between the reference coefficient $\lambda_{r}$ and the actual one 
$\lambda_{a}$.

For PID parameter tuning, we chose to use the open loop Ziegler-Nichols rules to individuate starting point values, which were heuristically adjusted to achieve better control results. In this case, we obtained the best performance with a PID $(P=1.5, I=4000$ and $D=0)$ control for all the $\lambda_{r}$ range $\left(5.8 \times 10^{-4} \leq \lambda \leq 3 \times 10^{-3}\left[\mathrm{~N} / \mathrm{mm}^{3}\right]\right)$ that can be reproduced through FYD-2.

In fig. 13 the results of the control for $\lambda_{r}$ equal to $6.3 \times$ $10^{-4} \mathrm{~N} / \mathrm{mm}^{3}$ for SS3 are reported. We get a Root Mean Square Error (RMSE) of $2.2 \times 10^{-} 6 \mathrm{~N} / \mathrm{mm}^{3}$, corresponding to a Percentage Root Mean Squared Error (RMSEP) of $3.8 \%$.

\section{Human EXPERIMENTS}

To properly characterize FYD-2 performance in terms of its capability in eliciting a correct softness perception, we performed two main types of experiments with humans. In the first type of experiments, participants were asked to perform relative and absolute softness cognition tasks [23]: more specifically, in relative cognition tasks, participants were asked to touch three rendered silicone specimens, whose force-area behavior was artificially simulated using FYD-2, and to rank them on the basis of the perceived softness. In absolute softness recognition tasks, participants were asked to correctly associate the artificial silicone specimens with the real ones. In the second type of experiments, we characterized FYD-2 performance in terms of Just Noticeable Difference (JND) and Weber's fraction for different motor positions, and hence different profiles of stiffness. Finally, we briefly discuss an application of the FYD-2 suitably integrated into an experimental apparatus for perceptual experiments, where a change in the contact area spread rate was proven to induce an illusory percept of finger displacement, as reported in [31].

\subsection{Experiment Type 1: Cognition Tasks}

The first type of experiments (hereinafter referred to as Experiment Type 1) consists of two parts: relative cognition task and absolute cognition task. Each experiment type was performed by a different group of participants.

\subsubsection{Relative Cognition Task}

\begin{tabular}{|l||c|c|c|c|}
\hline & SH1 & SH2 & SH3 & Relative Accuracy \\
\hline SH1 & 65 & 4 & 1 & $92.9 \%$ \\
\hline SH2 & 4 & 66 & 0 & $94.3 \%$ \\
\hline SH3 & 1 & 0 & 60 & $98.5 \%$ \\
\hline
\end{tabular}

TABLE 1: Confusion Matrix for Relative Cognition Task.

Thirteen right-handed healthy participants (1 Female, Age: $24.86 \pm 3.16$, mean $\pm \mathrm{SD}$ ) gave their informed consent to participate to the experiment. No one had any physical limitation which would have affected the experimental outcomes. The experimental procedure was approved by the Ethical Committee of the University of Pisa. SH1, SH2 and SH3 were presented three times in a random order to the participants and then they were asked to probe and sort them in terms of softness. They were instructed to do so by pressing vertically or tapping the index finger of their dominant hand against the specimens, with a frequency of approximately $0.2 \mathrm{~Hz}$. They were recommended to not perform movements of the finger across the surface and not to apply lateral forces, thus avoiding any anisotropic or distortion effect in softness perception [5]. Participant arm was blocked within an arm holder, such that participants were able to use only their wrist and finger for softness probing of the objects. The device was covered with a black fabric drape, in order to eliminate any visual cue for participants and enable a proper usage of the device webcamera. Participants wore headphones with white noise, to prevent the usage of any auditory cue.

They did not have time limitations since they were allowed to touch the rendered specimens as many times as they wanted. The entire ranking experiment was repeated five times. Results of the ranking experiments are shown in Table 1, where perceived softness is reported versus artificially reproduced softness, with the relative accuracy in discrimination for each specimen. Values on the diagonal express the amount of correct answers. The average cognition rate is $95.2 \%$, close to $100 \%$ (chance level of $33.34 \%$ ), indicating that the device is able to render different stiffness levels, which are well recognizable by the participants. This result is comparable with the one reported in [23], outperforming performance reported for previous versions of the fabric yielding display and pneumatic CASR display [30].

\subsubsection{Absolute Cognition Task}

\begin{tabular}{|l||c|c|c||c|}
\hline & SH1 & SH2 & SH3 & Relative Accuracy \\
\hline \hline SS1 & 51 & 13 & 1 & $78.5 \%$ \\
\hline SS2 & 6 & 46 & 13 & $70.8 \%$ \\
\hline SS3 & 0 & 4 & 61 & $93.8 \%$ \\
\hline
\end{tabular}

TABLE 2: Confusion Matrix for Absolute Cognition Task.

Thirteen right-handed healthy participants (6 F, Age: $21.15 \pm 3.13$, mean $\pm \mathrm{SD}$ ) gave their informed consent to participate to the experiment. No one had any physical limitation which would have affected the experimental outcomes. The experimental procedure was approved by the Ethical Committee of the University of Pisa. The experimental apparatus was similar to the one used for the relative recognition tests. SH1, SH2 and SH3 were presented three times in a random order to participants and then they were asked to associate them to their physical counterparts SS1, SS2 and SS3. One rendered specimen was randomly presented at one time. Real specimens were positioned on a white sheet of paper on the desk near the arm stand, so that the participant did not have to do large excursions with the arm. The silicones were posed on numbered circles on the sheet in a random order for each participant. Participant arm was placed into the arm holder and she/he was required to consequently touch with the index of the dominant hand both the artificial and real specimens using the same techniques previously described. Participants did not have time limitations since they were allowed to touch the silicone specimens and the rendered stimulus and to go back and forth between them as many times as they wanted. 
Participants wore headphones with white noise, to prevent the usage of any auditory cue. Since the experiment was performed in blind conditions, participants were guided by the experimenter to touch the object and the device. Results of the ranking experiments are shown in Table 2, where the perception of artificial specimens was associated to the perception of real ones in a confusion matrix structure. Values on the diagonal express the amount of correct answers. The average cognitive rate is $81 \%$, chance level of $33.34 \%$. As one could expect the errors on the central silicone are higher, while $45 \%$ and $20 \%$ silicones are fairly more distinguishable. Results for absolute cognition tasks are comparable to the ones reported in literature for other softness devices [10], [23].

\subsection{Experiment 2: Psychophysical Characterization}

The second type of experiments (hereinafter referred to as Experiment Type 2) aimed at determining JND of the FYD-2 by using the method of constant stimuli [39]. More specifically, the JND was defined in terms of the just noticeable difference in motor position, which is able to elicit a different softness perception. Knowing the JND was then possible to determine the associated Weber's fraction. The experimental apparatus was similar to the one described for Experiment Type 1. Participants were asked to touch and probe for softness using the index finger of their dominant hand the surface of the device, at different positions of the motor corresponding to different stiffness characteristics $(\theta)$. They experienced paired stimuli and they were asked to indicate which stimulus in the pair was the softer one. The inter-stimuli interval was $1 \mathrm{~s}$ while stimulus exploration lasted $3 \mathrm{~s}$. Each pair consisted of a reference stimulus (RS) of $50^{\circ}$ and a comparison stimulus (CS), presented in random order. We used five discrete and equally spaced motor positions between 10 and $90^{\circ}$. The minimum and maximum levels were chosen in a preliminary study such that they were almost always judged as less than or greater than RS. During the experiment 100 couples of stimuli were presented to each participant, in a randomized order. All the answers of the participants were saved in a file for future analyses. Twelve right handed, healthy participants (7 F, Age; $22.83 \pm 1.95$, mean $\pm \mathrm{SD}$ ) gave their informed consent to participate to the experiment. No one had any physical limitation that would have affected the experimental outcomes. The binomial responses of the participants were fitted using Generalized Linear Mixed Models (GLMMs).

\subsubsection{Results}

We used GLMMs to determine JND and Point of Subjective Equality (PSE) through all the participants [40] ${ }^{1}$. Indeed, in GLMMs the overall variability of the data sets consists of two different components: a fixed and a random component. The fixed component takes into account and estimates the effect of interest, i.e. the experimental effect. In this manner we can estimate a single model across all the participants, but allowing different variability and sample size for each

1. For further details, the reader may refer to: http://mixedpsychophysics. wordpress.com/ participant. Therefore, the expected value of the response can be expressed as:

$$
\Phi^{-1}\left[P\left(Y_{i j}=1\right)\right] \sim \beta_{0}+\beta_{1} x_{i j}
$$

where $\Phi^{-1}$ is the logit transformation, which relates the response variable to the linear predictor; $Y_{i j}$ is the response variable for subject $i$ at trial $j$ and $\left[P\left(Y_{i j}=1\right)\right]$ is the probability of a larger response, i.e. it has the value 1 if the participant reported that the CS was softer than RS (and 0 otherwise); $x_{i j}$ is the explanatory variable, i.e. the motor angular position. $\beta_{0}$ and $\beta_{1}$ are the fixed effect parameters, i.e. the intercept and the slope of the linear function (linear predictor), which are the same for all the subjects. The point of subjective equality (PSE) is

$$
P S E=-\frac{\beta_{0}}{\beta_{1}}
$$

The random component can estimate the heterogeneity between subjects. For further details the reader may refer to [40], [41], [42].

We considered two GLMM models for fitting, where the fixed effect (motor position) was the same as well as the used link function (logit function): (1) in the first model we considered only a random intercept, while in the second model (2), we considered random intercept, random slope and the correlation of the two. In other terms, we assume that in each different participant, the response has a different distribution. More specifically, in model (1) we assume that the response distribution of different participants change only for the intercept value - that is, all the underling psychometric functions of the participant are shifted one to the other, but they have the same slope. In model (2), we assume that different participants have also different slopes.

A maximum likelihood (ML) estimation was used to find the coefficients of the models, solved numerically using the Gauss-Hermite quadrature implemented in the function glmer in $\mathbf{R}$ package $\operatorname{lm} \mathrm{4}^{2}$.

In order to find which model fits best with data, there are different procedures for model comparison. The Likelihood Ratio test (LR) and the Akaike Information Criterion (AIC) [40] are common procedures for model comparison. (Note: lower AIC values indicate better fits). Likelihood Ratio test (LR) reveals significant differences $\left(p<10^{-10}\right)$ between the fit of the two models (1) and (2) described above. Furthermore, model (2) exhibits the smallest value for the Akaike Information Criterion (AIC) (242 vs. 446).

These observations lead us to use model (2) for fitting psychometric function and to take into account between subject variability.

What we found is a PSE of $51.35 \pm 1.40$ (standard error) $\left[^{\circ}\right]$, which indicates an average Constant Error (i.e. a difference between point of objective equality and PSE) less than $2^{\circ}$. Accordingly, we found JND value of $12 \pm 2.19$ (standard error) $\left.{ }^{\circ}\right]$. The Weber's fraction can be obtained from JND, dividing it for the RS. What we found is a Weber fraction of $24 \%$,

2. The R Project for Statistical Computing - www.r-project.org; package "Ime4: Linear mixed-effects models using s4 classes. $r$ package version 0.999375-39” 2011. D. Bates, M. Maechler, and B. Bolker 
which is comparable to the values of Weber's fraction obtained in softness discrimination tasks with real silicone blocks [8].

\subsection{Related Perceptual Experiment}

In [31], the FYD-2 was used to investigate the illusion induced in finger displacement perception by the change of the contact area on the user finger pad. The hypothesis was that when the compliance of the contacted object unexpectedly changes, the perceptual system can misestimate the indentation of the finger into the object and hence the position of the finger. In the experiments reported [31], the FYD-2 was integrated into an experimental apparatus. More specifically, it was placed on a lift and participants compared the passive displacement of the index finger between a reference and a comparison stimulus. The compliance of the contacted object changed unexpectedly between the two stimuli, corresponding to different motor angular positions, thus producing different area-displacement relationships. What we found is that in accordance with the tested hypothesis, the modulation of the area-displacement relationship produced a bias in the perceived displacement of the finger.

\section{Conclusions}

In this paper we have presented the design and psychophysical characterization of a fabric-yielding softness display, FYD2. The device enables to on-line measure and control the area at contact between the finger and the surface of the fabric, thus reproducing CASR curves [10] by regulating the stretching state of the fabric. FYD-2 was proven to be able to effectively reproduce softness characteristics of real and artificial specimens and to elicit correct softness perception in human users, with performance greater or similar to the ones obtained with other softness displays and real objects. Future works will aim at investigating how we can exploit the "translational" degree of freedom of FYD-2 to provide the users with a more immersive haptic experience. At the same time, we will work on making this device wearable and multi-fingered.

\section{ACKNOWLEDGMENTS}

This work is supported in part by the European Research Council under the Advanced Grant SoftHands "A Theory of Soft Synergies for a New Generation of Artificial Hands" (no. ERC-291166), and by the EU FP7 project (no. 601165) "WEARable HAPtics for Humans and Robots (WEARHAP)". Authors wish to thank Omar Al Atassi and Simone Fani for their support in performing experiments.

\section{REFERENCES}

[1] W. Tiest and A. M. L. Kappers, "Cues for haptic perception of compliance," Haptics, IEEE Transactions on, vol. 2, no. 4, pp. 189-199, Oct 2009.

[2] K. Hoyt, B. Castaneda, M. Zhang, P. Nigwekar, P. A. di Sant'Agnese, J. V. Joseph, J. Strang, D. J. Rubens, and K. J. Parker, "Tissue elasticity properties as biomarkers for prostate cancer," Cancer Biomarkers, vol. 4, no. 4, pp. 213-225, 2008.
[3] F. Avanzini and D. Rocchesso, "Controlling material properties in physical models of sounding objects," in in Proc. Int. Computer Music Conf., La Habana, 2001, pp. 91-94.

[4] V. Varadharajan, R. Klatzky, B. Unger, R. Swendsen, and R. Hollis, "Haptic rendering and psychophysical evaluation of a virtual threedimensional helical spring," in Haptic interfaces for virtual environment and teleoperator systems, 2008. haptics 2008. symposium on. IEEE, 2008 , pp. 57-64

[5] S. J. Lederman and R. L. Klatzky, "Relative availability of surface and object properties during early haptic processing." Journal of Experimental Psychology: Human perception and performance, vol. 23, no. 6, p. 1680, 1997.

[6] R. L. Klatzky, S. J. Lederman, and D. E. Matula, "Imagined haptic exploration in judgments of object properties." Journal of experimental psychology: learning, memory, and cognition, vol. 17, no. 2, p. 314, 1991.

[7] H. Z. Tan, N. I. Durlach, G. L. Beauregard, and M. A. Srinivasan, "Manual discrimination of compliance using active pinch grasp: The roles of force and work cues," Perception \& psychophysics, vol. 57, no. 4, pp. 495-510, 1995.

[8] F. K. Freyberger and B. Färber, "Compliance discrimination of deformable objects by squeezing with one and two fingers," in Proc. EuroHaptics, vol. 2006, 2006, pp. 271-276.

[9] M. A. Srinivasan and R. H. LaMotte, "Tactual discrimination of softness: abilities and mechanisms," in Somesthesis and the Neurobiology of the Somatosensory Cortex. Springer, 1996, pp. 123-135.

[10] A. Bicchi, D. E. De Rossi, and E. P. Scilingo, "The role of the contact area spread rate in haptic discrimination of softness," IEEE trans. on Robotics and Automation, vol. 16, no. 5, pp. 496-504, October 2000.

[11] S. Yazdian, A. J. Doxon, D. E. Johnson, H. Z. Tan, and W. R. Provancher, "Compliance display using a tilting-plate tactile feedback device," in Haptics Symposium (HAPTICS), 2014 IEEE. IEEE, 2014, pp. 13-18.

[12] E. P. Scilingo, M. Bianchi, G. Grioli, and A. Bicchi, "Rendering softness Integration of kinesthetic and cutaneous information in a haptic device," Haptics, IEEE Transactions on, vol. 3, no. 2, pp. 109-118, 2010.

[13] A. Song, J. Liu, and J. Wu, "Softness haptic display device for humancomputer interaction," Human Computer Interaction, InTech, pp. 257-277, 2008.

[14] M. Konyo, S. Tadokoro, and T. Takamori, "Artificial tactile feel display using soft gel actuators," in Robotics and Automation, 2000. Proceedings. ICRA'OO. IEEE International Conference on, vol. 4. IEEE, 2000, pp. 3416-3421.

[15] C. Mavroidis, C. Pfeiffer, J. Celestino, and Y. Bar-Cohen, "Controlled compliance haptic interface using electrorheological fluids," in SPIE's 7th Annual International Symposium on Smart Structures and Materials. International Society for Optics and Photonics, 2000, pp. 300-310.

[16] A. Bicchi, E. P. Scilingo, N. Sgambelluri, and D. De Rossi, "Haptic interfaces based on magnetorheological fluids," in Proceedings 2th International Conference Eurohaptics 2002, 2002, pp. 6-11.

[17] G. Moy, C. Wagner, and R. Fearing, "A compliant tactile display for teletaction," in Robotics and Automation, 2000. Proceedings. ICRA '00. IEEE International Conference on, vol. 4, 2000, pp. 3409-3415 vol.4.

[18] M. Takaiwa and T. Noritsugu, "Development of pneumatic human interface and its application for compliance display," in Industrial Electronics Society, 2000. IECON 2000. 26th Annual Confjerence of the IEEE, vol. 2, 2000, pp. 806-811 vol.2.

[19] L. B. Porquis, D. Maemori, N. Nagaya, M. Konyo, and S. Tadokoro, "Presenting virtual stiffness by modulating the perceived force profile with suction pressure," in Haptics Symposium (HAPTICS), 2014 IEEE. IEEE, 2014, pp. 289-294.

[20] V. Hayward, "Is there a "plenhaptic" function?" Phil. Trans. R. Soc. B, vol. 366, pp. 3115-3122, 2011.

[21] A. Bicchi, M. Gabiccini, and M. Santello, "Modelling natural and artificial hands with synergies," Philosophical Transactions of the Royal Society B: Biological Sciences, vol. 366, no. 1581, pp. 3153-3161, 2011.

[22] M. Bianchi, "On the role of haptic synergies in modelling the sense of touch and in designing arti cial haptic systems," Ph.D. dissertation, 2012.

[23] K. Fujita and H. Ohmori, "A new softness display interface by dynamic fingertip contact area control," in World Multiconf. on Systemics, Cybernetics and Informatics, Orlando, Florida (USA), July 2001, pp. 78-82.

[24] H. Yokota, A. Yamamoto, H. Yamamoto, and T. Higuchi, "Producing softness sensation on an electrostatic texture display for rendering diverse tactile feelings," in EuroHaptics Conference, 2007 and Symposium on Haptic Interfaces for Virtual Environment and Teleoperator Systems. World Haptics 2007. Second Joint. IEEE, 2007, pp. 584-585.

[25] F. Kimura, A. Yamamoto, and T. Higuchi, "Development of a contact width sensor for tactile tele-presentation of softness," in Robot and 
Human Interactive Communication, 2009. RO-MAN 2009. The 18th IEEE International Symposium on, Sept 2009, pp. 34-39.

[26] — "Development of a 2-dof softness feeling display for tactile telepresentation of deformable surfaces," in Robotics and Automation (ICRA), 2010 IEEE International Conference on, May 2010, pp. 1822-1827.

[27] F. Kimura and A. Yamamoto, "Rendering variable-sized lump sensations on a softness tactile display," in World Haptics Conference (WHC), 2013. IEEE, 2013, pp. 97-102.

[28] J. C. Gwilliam, A. Degirmenci, M. Bianchi, and A. M. Okamura, "Design and control of an air-jet lump display," in Proc. IEEE Haptics Symposium, 2012, pp. 45-49.

[29] M. Bianchi, E. P. Scilingo, A. Serio, and A. Bicchi, "A new softness display based on bi-elastic fabric," World Haptics Conference, pp. 382383, 2009.

[30] M. Bianchi, A. Serio, E. P. Scilingo, and A. Bicchi, "A new fabric-based softness display," in Proc. IEEE Haptics Symposium, 2010, pp. 105 -112.

[31] A. Moscatelli, M. Bianchi, A. Serio, O. Al Atassi, S. Fani, A. Terekhov, V. Hayward, M. Ernst, and A. Bicchi, "A change in the fingertip contact area induces an illusory displacement of the finger," in Haptics: Neuroscience, Devices, Modeling, and Applications, ser. Lecture Notes in Computer Science, M. Auvray and C. Duriez, Eds. Springer Berlin Heidelberg, 2014, pp. 72-79.

[32] A. Serio, M. Bianchi, and A. Bicchi, "A device for mimicking the contact force/contact area relationship of different materials with applications to softness rendering," in Intelligent Robots and Systems (IROS), 2013 IEEE/RSJ International Conference on, Nov 2013, pp. 4484-4490.

[33] M. Bianchi, A. Serio, E. Scilingo, and A. Bicchi, "A fabric-based approach for softness rendering," in Multisensory Softness, ser. Springer Series on Touch and Haptic Systems, M. Di Luca, Ed. Springer London, 2014, pp. 219-240.

[34] E. Scilingo, N. Sgambelluri, G. Tonietti, and A. Bicchi, "Integrating two haptic devices for performance enhancement," in EuroHaptics Conference, 2007 and Symposium on Haptic Interfaces for Virtual Environment and Teleoperator Systems. World Haptics 2007. Second Joint, March 2007, pp. $139-144$.

[35] "3-d finite-element models of human and monkey fingertips to investigate the mechanics of tactile sense," ASME, J. of Biomech. Eng., vol. 125, pp. 682-691, 2003.

[36] G. J. Gerling, "Sa-i mechanoreceptor position in fingertip skin may impact sensitivity to edge stimuli," Applied Bionics and Biomechanics, vol. 7, no. 1, pp. $19-29,2010$.

[37] B. K. P. Horn and B. G. Schunk, "Determining optical flow," Artificial Intelligence, vol. 17, pp. 185-203, 1981.

[38] B. Hannaford and A. M. Okamura, Springer Handbook on Robotics. Heidelberg, Germany: Springer, 2008, ch. Haptics, pp. 719-739.

[39] G. A. Gescheider, Psychophysics: Method, Theory, and Application. Hillsdale, NJ: Lawrence Erlbaum Associates, Inc., 1985.

[40] A. Moscatelli, M. Mezzetti, and F. Lacquaniti, "Modeling psychophysical data at the population-level: The generalized linear mixed model," Journal of vision, vol. 12, no. 11, p. 26, 2012.

[41] B. M. Bolker, M. E. Brooks, C. J. Clark, S. W. Geange, J. R. Poulsen, M. H. H. Stevens, and J.-S. S. White, "Generalized linear mixed models: a practical guide for ecology and evolution," Trends in ecology \& evolution, vol. 24, no. 3, pp. 127-135, 2009.

[42] A. Ajoudani, S. Godfrey, M. Bianchi, M. Catalano, G. Grioli, N. Tsagarakis, and A. Bicchi, "Exploring teleimpedance and tactile feedback for intuitive control of the pisa/iit softhand," Haptics, IEEE Transactions on, vol. 7, no. 2, pp. 203-215, April 2014.

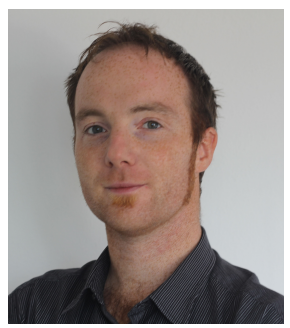

Matteo Bianchi Matteo Bianchi received the B.S degree cum laude and the M.S cum laude in Biomedical Engineering from Università di Pisa, Italy, in 2004 and 2007, respectively and the PhD in Automatics, Robotics and Bioengineering from Università di Pisa in 2012. Currently, he is working as a Post-Doctoral researcher at the Advanced Robotics Department (ADVR) of Istituto Italiano di Tecnologia (IIT) and as a collaborator of Centro di Ricerca "E. Piaggio" of Università di Pisa. From January to June 2011, he worked as visiting student at the Laboratory for Computational Sensing and Robotics at Johns Hopkins University, Baltimore, MD. His research interests include haptic interface design, control and validation with applications in virtual reality, robotics/medical robotics (robot-assisted minimally invasive surgery and prosthetics) and assisitve/affective human-robot interaction; human and robotic hands: optimal sensing and control; psychophysics and mathematical modelling of the sense of touch and human manipulation. He is an author of contributions to international conferences and journals. In 2010 he was finalist for the Best Student Paper and Best Paper Award at the IEEE Haptics Symposium in Boston (US - MA); in 2012 he was awarded with the JCTF novel technology paper award at the IEEE/RSJ IROS Conference in Villamoura, Portugal and in 2014 with the best paper award (poster category) at the Eurohaptics Conference in Versailles, France. He is a member of the IEEE.

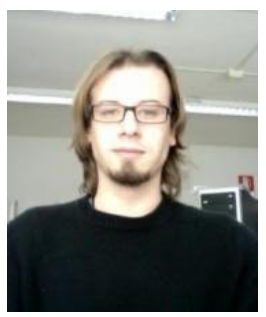

Alessandro Serio Alessandro Serio received the Automation Engineering degree and the $\mathrm{PhD}$ degree in Robotics, Automatics and Bioengineering both from Università di Pisa, Italy, in 2009 and 2013, respectively. Currently he holds a Post-Doc position at the Research Center "E. Piaggio" of the Università di Pisa. His main research topics are hardware and software design of robotic hands, tactile perception, haptics and robot sensing. He is member of the IEEE. 